\title{
Qualität und Innovation in der Gesundheitsversorgung
}

\section{Lea Muntwyler}

Mitarbeiterin Kommunikation/Marketing der SGAIM

\author{
Am 2. Health Symposium spannte die SGAIM mit dem Nationalen Forschungspro- \\ gramm (NFP) 74 des Schweizerischen Nationalfonds zusammen. Sie stellten im \\ neueröffneten Casino Bern die Themen Qualität und Innovation in der Gesund- \\ heitsversorgung ins Zentrum - mit Erfolg.
}

Von der Kommunikation bei Patienten mit Impfskepsis, der Schliessung von Hausarztpraxen über die Dauer von Antibiotikatherapien: Akteure aus Medizin, Politik und Wirtschaft referierten und diskutierten am exklusiven Event über aktuelle Themen in der Gesundheitsversorgung. Dabei tauschten sich die Fachleute über aktuelle Herausforderungen und innovative Tools aus der Praxis aus.

SGAIM-Co-Präsident Drahomir Aujesky und NFP74-Präsident Milo Puhan eröffneten gemeinsam den Anlass. Dieser konnte nicht zu einem spannenderen Zeitpunkt stattfinden: Gleich mehrere Projekte des NFP 74 stehen kurz vor dem Abschluss - die Teilnehmenden kamen so in den Genuss, über neuste Forschungsprojekte und sogar erste Ergebnisse informiert zu werden. Dass die Themen des Health Sympo-

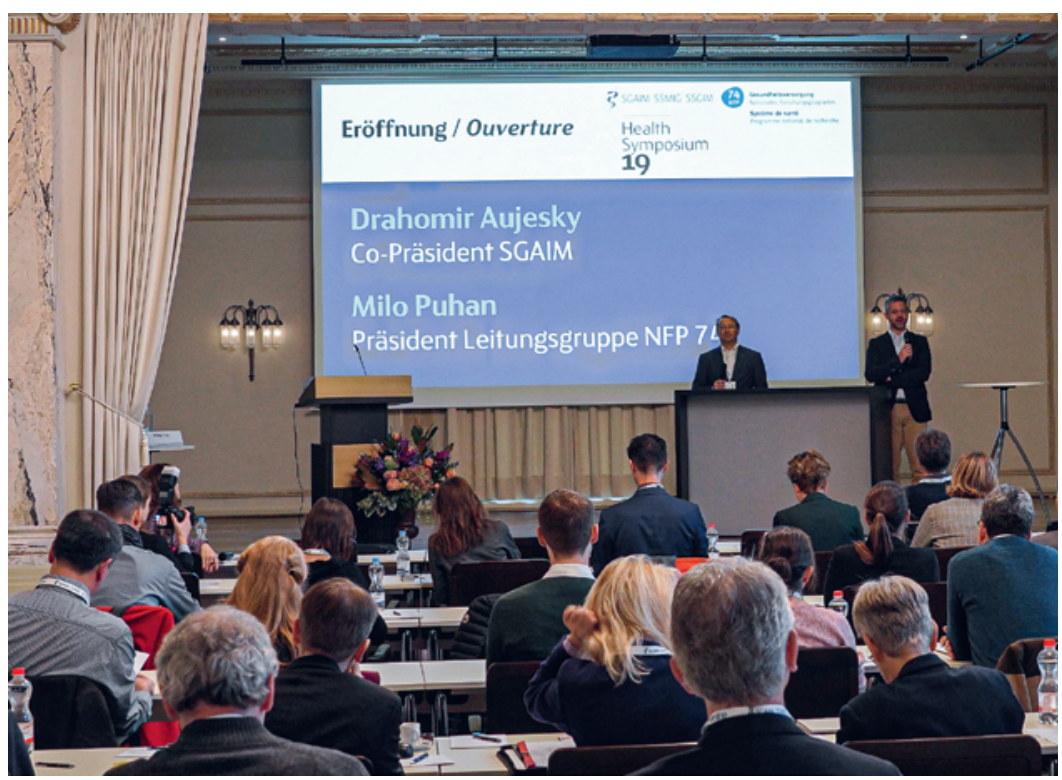

Machten gemeinsame Sache: SGAIM-Co-Präsident Drahomir Aujesky und NFP-74-Präsident Milo Puhan. siums 2019 hochaktuell sind, entging auch der SRF-Moderatorin Marina Villa nicht, die souverän durch die Veranstaltung führte.

\section{Mehr ist nicht immer besser}

Das Auftaktreferat hielt Maria Wertli über die Faktoren, welche die Durchführung von Wahleingriffen in der Schweiz beeinflussen. Sie stellte dazu neueste Zahlen vor und machte klar, dass die Durchführung von Eingriffen auch durch die persönlichen Einstellungen der Ärzteschaft beeinflusst wird. Mithilfe von Daten des Bundesamts für Statistik und der FMH erstellte sie eine Karte der Spitalversorgungsgebiete, die zeigt, wie häufig Hüft- und Kniegelenkersatz, operative Entfernungen der Gebärmutter oder Prostata, Wirbelsäulenchirurgie und bariatrische Eingriffe in welchen Spitälern und Regionen durchgeführt werden. Die Karte zeigte Verblüffendes: Je nach Spital oder Region wird ein Eingriff durchgeführt oder nicht. Wertli führte dies auf sozio-demographische Gründe zurück.

\section{Wieso zögern Eltern und Mediziner zu impfen?}

Der hochaktuellen Frage zur Impfskepsis ging Philip Tarr vom Kantonsspital Baselland nach. Sein Forschungsprojekt zielt darauf $a b$, die Gründe für die Impfskepsis von Eltern und der Ärzteschaft kennenzulernen, indem Gespräche mit diesen Gruppen gesucht werden, um mit den Erkenntnissen die Kommunikationsinstrumente für die Ärzteschaft zu verbessern. Tarr gab dazu auch praxisnahe Tipps ab: Paradoxerweise würden Wiederholungen von Fakten sowie die Betonung der Vorteile von Impfungen zu Misstrauen und Skepsis führen. Angstmacherei sei auch kontraproduktiv. Dabei warnte er, dass die grosse Mehrheit 
der Impfskeptikerinnen und -skeptiker schliesslich doch Impfungen durchführen (lassen): «Nur 1-3\% der Schweizer Bevölkerung ist strikt gegen alle Impfungen", sagte Tarr. Sein Fazit nach rund 90\% der Interviews: Eine persönliche, patientenorientierte Behandlung sowie reduzierte Zugangshürden von Kantonen könnten die Impfrate in der Schweiz erhöhen.

\section{Wenn Hausarztpraxen schliessen ...}

Nach der Kaffeepause referierte Michael Gerfin über die Auswirkungen der Schliessung von Hausarztpraxen auf Patientinnen und Patienten und das Gesundheitswesen. Er kam zum Schluss, dass die Schliessung von Hausarztpraxen das Verhalten von Patientinnen und Patienten erheblich beeinflusst. Diese würden weniger oft Ärzte konsultieren: «Über $75 \%$ von Patientinnen und Patienten in diesen Regionen ersetzen die Arztkonsultationen nicht!» Andere würden häufiger Spezialisten oder ambulante Krankenhausabteilungen aufsuchen. "Dabei sind Patientinnen und Patienten in Regionen mit einer niedrigen Arztpraxendichte stärker betroffen, weil sich die Suche nach einer Ersatzhausarztpraxis schwierig gestaltet», sagte der Experte vom Volkswirtschaftlichen Institut der Universität Bern.

\section{Kampf der Antibiotika-Fehl- und -Überversorgung}

Wie lässt sich die Dauer einer Antibiotikatherapie zuverlässig festlegen? Dieser brisanten Frage ging Angela Huttner von den Hôpitaux Universitaires de Genève (HUG) in ihrem Vortrag zum «Pirate Project» nach. Dieses interdisziplinäre Projekt berechnet eine optimale und individualisierte Antibiotikatherapie. Obwohl die Resultate erst im Frühjahr veröffentlicht werden, habe das Projekt erfreuliche Resultate vorzuweisen, verriet Huttner.

Im nächsten Referat stellte Agnė Ulytė von der Universität Zürich eine Studie vor, die den Einfluss von Guidelines und Empfehlungen auf die medizinische Behandlung untersuchte.

\section{NFP 74 Gesundheitsversorgung}

Das Nationale Forschungsprogramm 74 "Gesundheitsversorgung" des Schweizerischen Nationalfonds will einen Beitrag zu «Smarter Health Care» leisten. Ziel ist es, Erkenntnisse zu gewinnen, wie die Versorgung von chronisch kranken und multimorbiden Menschen verbessert werden kann. Zudem sollen längerfristig die Verfügbarkeit, Zugänglichkeit und Verknüpfung von Gesundheitsdaten optimiert werden. Schliesslich soll ein Beitrag zum Aufbau einer starken Forschungsgemeinschaft geleistet werden, die weltweit führende Versorgungsforschung betreibt. www.nfp74.ch

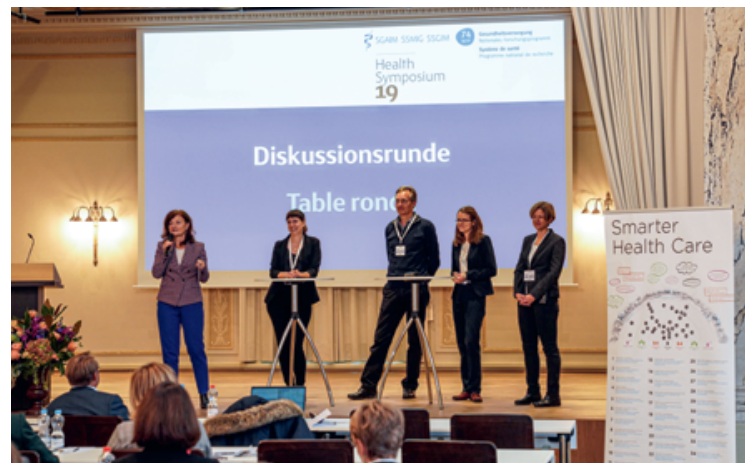

Die Moderatorin Marina Villa leitete die Diskussionen zwischen den Referierenden und dem Publikum.

\section{Interprofessionalität als Schlüssel}

Beat Müller, Kantonsspital Aarau, stellte das Projekt InHospiTOOL vor und zeigte anhand einer Studie von 30000 Follow ups, wie die systematische interprofessionelle Zusammenarbeit die Aufenthaltsdauer im Spital beeinflusst. In seinem Fazit plädierte Müller für alternative, individualisierte Zugänge bei der Betreuung von Patientinnen und Patienten im Spital.

\section{Lobbyismus $1 \mathrm{x} 1$}

Danach führte Céline Mavrot aus, wie gesundheitspolitische Anliegen bei Entscheidungsträgerinnen und -trägern tatsächlich ankommen. Je nach Zielgruppe, Zeitpunkt, Kontext und Absicht sei eine andere Strategie nötig, so die Expertin.

\section{Qualität ist kein Zufall}

Im letzten Vortrag sprach erneut Maria Wertli - diesmal in ihrer Funktion als Kommissionspräsidentin Qualität der SGAIM. Sie stellte die neue Qualitätsstrategie vor, die beabsichtigt, die SGAIM als einen bedeutenden nationalen Player innerhalb der Ärzteschaft zu positionieren. In den Genuss davon kommen auch die SGAIM-Mitglieder: Künftig sollen Hilfsmittel für die Praxis auf der Webseite zu finden sein.

Der Anlass im Zeichen der Qualität und Innovation kam bei den Teilnehmenden gut an: «Ich fand das Health Symposium sehr nützlich. Insbesondere die Bedeutung der interdisziplinären und interprofessionellen Zusammenarbeit zum Wohle der Patientinnen und Patienten sowie die Kommunikationstipps werden mich in meiner Tätigkeit begleiten", meinte ein Besucher. Auch die Umfrage nach dem Event zeigte: Das Health Symposium 2019 leistete einen Beitrag zur Diskussion aktueller Themen der Gesundheitsversorgung, wobei Diskurs und Vernetzung nicht zu kurz kamen. 